\title{
La creatividad que se experiencia
}

\author{
Ingold, Tim \\ The School of Social Science, University of Aberdeen, Scotland
}

\begin{abstract}
Este artículo se publicó en su version original en inglés en Pragmatics \& Cognition 22:1 (2014) - Pages: 124-139. John Benjamins Publishing Company. Amsterdam/Philadelphia. ${ }^{1}$

La traducción se ha realizado con permiso del autor y del editor de la publicación original.

La traducción se ha realizado dentro del Grupo de Investigación de la Universidad de Alicante Proyectos Arquitectónicos: Pedagogías Críticas, Políticas Ecológicas y Prácticas Materiales.

El equipo ha estado coordinado por Ester Gisbert Alemany y han participado Joaquín García Marín, Jose Carrasco y Enrique Nieto.
\end{abstract}

\section{Resumen}

En muchas ocasiones se presenta la creatividad como un factor enigmático que explica la emergencia espontánea de lo radicalmente nuevo. La obsesión con la novedad supone, sin embargo, un enfoque hacia los productos finales y una atribución retrospectiva de sus formas a ideas sin precedentes surgidas en la mente de los individuos. Esta obsesión impide reconocer el potencial de generación formal de las relaciones y procesos en los que cosas y personas se hacen y crecen. En estos procesos se acostumbra a pedir a los practicantes que copien las obras de maestros pasados. A pesar de dejarse guiar por un guión o partitura al hacerlo, cada individuo ha de improvisar su propio camino a través de la variedad de tareas que supone cualquier práctica. Con ejemplos extraídos de los ámbitos de la música, la caligrafía y el encaje de bolillos, muestro que las fuentes de la creatividad no residen en la cabeza de la gente, sino en su atender a un mundo en constante formación. Para este tipo de creatividad, experienciada en lugar de hecha, la imaginación no consiste tanto en la capacidad de topar con nuevas ideas como en el impulso aspiracional de una vida que no es únicamente vivida, sino también guiada. Pero hacia un destino aún no fijado. Al abrirse a lo desconocido, al resultar expuesta, la imaginación no guía a través de la maestría, sino de la entrega. Por tanto la creatividad que se experiencia, la de la acción sin agencia, es la de la vida misma.

Palabras clave: Aspiración, atender, caligrafía, "concrescencia", copia, creatividad, hacer, educación, historia, imaginación, improvisación, innovación, encaje, música, notación, ontogénesis, ejecución, tiempo, experienciar.

\begin{abstract}
Creativity is often portrayed as an X-factor that accounts for the spontaneous generation of the absolutely new. Yet the obsession with novelty implies a focus on final products and a retrospective attribution of their forms to unprecedented ideas in the minds of individuals, at the expense of any recognition of the form-generating potentials of the relations and processes in which persons and things are made and grown. In these processes, practitioners are characteristically called upon to copy the works of past masters. However, though they may be guided by a script or score, every practitioner has to improvise his or her own passage through the array of tasks the performance entails. With examples from music, calligraphy and lace-making, I show that the wellsprings of creativity lie not inside people's heads, but in their attending upon a world in formation. In this kind of creativity, undergone rather than done, imagination is not so much the capacity to come up with new ideas as the aspirational impulse of a life that is not just lived but led. But where it leads is not yet given. In opening to the unknown - in exposure - imagination leads not by mastery but by submission. Thus the creativity of undergoing, of action without agency, is that of life itself.
\end{abstract}

Keywords: Aspiration, attention, calligraphy, concrescence, copying, creativity, doing, education, history, imagination, improvisation, innovation, lace-making, music, notation, ontogenesis, performance, time, undergoing

1 https://www.benjamins.com/\#catalog/journals/pc.22.1/main 


\section{Tiempo, creatividad e historia.}

Los psicólogos tienen una concepción singular de la creatividad. Tienden a pensar que es un factor enigmático que, en algún lugar del cerebro, explica la generación espontánea de lo absolutamente novedoso. Una suerte de bujía que enciende la chispa en el motor mental y que aparentemente algunos individuos poseen y otros no. Por ello, se han dedicado grandes esfuerzos a descubrir este factor enigmático. Sin él, dicen, no habría habido historia, ni artes, ni cultura. Los humanos, como otros animales, hubieran estado anclados donde siempre han estado, haciendo lo que siempre han hecho, confinados en el lento avanzar de la evolución biológica.

Para ejemplificar esta tendencia nada mejor que regresar a los escritos de la filósofa y científica cognitiva Margaret Boden. Ella nos cuenta que existen unas cosas que se denominan «ideas creativas» y que literalmente surgen de la nada. Pero hay dos tipos de ideas creativas, las creativas-históricas y las creativas-psicológicas, en adelante creativas $\mathrm{H}$ y creativas $\mathrm{P}$. Una idea es creativa $\mathrm{P}$ si es nueva con respecto al individuo que la concibió. La idea es creativa $\mathrm{H}$ cuando es novedosa con respecto a la historia de toda la humanidad (Boden 1990:32). Cada episodio creativo $\mathrm{H}$, aludiendo al mantra de la exploración lunar, es «un paso pequeño para el hombre, pero un salto gigante para la humanidad». Este tipo de acontecimientos, cuando suceden, marcan las etapas de la historia, forjadas por genios excepcionales, mientras que todos los episodios creativos del tipo P permiten al resto de los mortales ponernos al día recapitulando las mismas etapas por nuestra cuenta.

La paradoja de la creatividad $\mathrm{H}$ es que al tiempo que tiene un carácter histórico, no tiene lugar en la historia. Por el contrario, lo que surge en la mente de un individuo creativo $\mathrm{H}$ estaría desprovisto de cualquier influencia histórica. Para Boden y para muchos otros psicólogos, la mente estaría completamente fuera de la historia y, en consecuencia, la historia en sí misma poco tendría que ver con la generación de ideas, sino con su reconocimiento y diseminación. En el proceso de generación de ideas creativas parece que cada mente está sola, desconectada del resto de personas, cosas y relaciones entre las que necesariamente opera. Tal y como enuncia de manera categórica Boden, «las creaciones de la mente deben producirse con los recursos mentales» (1990:29). Únicamente después de ser creadas las ideas entran en la historia (Ingold y Hallam 2007: 8-9).

Entonces ¿qué es la historia? Sólo puede ser un registro de innovaciones. ¿Y cómo saber si algo es o no innovador? Comparándolo con todo lo que sucedió anteriormente. Supongamos que se me ocurre una idea y quiero saber si es histórica o no. La única manera de averiguarlo es llevar a cabo un sondeo para comprobar si alguien la tuvo con anterioridad. La probabilidad de que sea nueva es de uno entre un millón. Así que nada, no hago historia. Sólo más de lo mismo, una vuelta más a la rueda de la reinvención perpetua. Por un momento había pensado que podría ser una idea histórica, que era genuinamente inédita, pero no, era sólo una idea psicológica. Ahora bien, supongamos que en mis búsquedas encuentro que nadie antes había reparado en eso. Entonces claramente mi idea ha hecho historia, al menos potencialmente, puesto que no ha tenido la oportunidad de entrar en la historia salvo que sea comunicada a otros y añadida al repertorio de ideas que ya están en circulación.

Desde esta perspectiva, que está ampliamente asumida aunque de una manera más implícita que explícita, los sucesos son históricos si no se repiten, esto es, si no pueden ser reducidos a reiteraciones de lo que ya ha sucedido antes. En este sentido los acontecimientos históricos son análogos a los números primos. Esta analogía fue propuesta por el historiador del arte George Kubler (1962:39), en un ensayo sobre historia y tiempo firmado hace unos cincuenta años. Según Kubler el tiempo está pautado por la repetición de sucesos, ante los que la historia se erige como la incidencia de lo único. En sus propias palabras:

«Nuestra percepción del tiempo depende de los sucesos regularmente recurrentes, al contrario que nuestra conciencia histórica, que depende del cambio y la variedad impredecibles. Sin cambio no hay historia, sin regularidad no hay tiempo. Tiempo e historia están relacionados como norma y variación: el tiempo es el escenario habitual de los caprichos de la historia» (Kubler 1962:71-72).

Por este motivo es habitual que los historiadores del arte estén tan entrenados en la cuestión sobre los originales y las copias. Algo es novedoso, es histórico, sólo cuando hace su primera aparición. Con las subsiguientes iteraciones la novedad desaparece. Los originales pertenecen a la historia pero las copias no, porque se acomodan en el tiempo. De hecho, es habitual que hagamos juicios similares sobre las personas. Consideramos que «la gente mayor» pertenece al tiempo y no a la historia: celebramos sus cumpleaños pero no esperamos que hagan nada relevante, incluso en contextos propicios a ello. Únicamente cuando disfrutan de la plenitud de la vida pueden hacer historia y ser singulares (como lo son los números primos). 


\section{Hacer y experienciar}

Sin embargo, algo fundamental se nos escapa con esta explicación: algo en lo que el tiempo, la creatividad y la historia se funden en uno. Encontré una enunciación seminal de este algo en el trabajo del teólogo americano Henry Nelson Wieman, quien, al igual que Kubler, escribía hace medio siglo. Para Wieman, como para Boden, existen dos tipos de creatividad, y es importante distinguirlas (Wieman 1961:63-66). Pero la distinción de Wieman es bastante diferente. En un primer sentido, dice, la creatividad es una actividad característica de los humanos. Un ser humano es creativo en este sentido "cuando construye algo según un nuevo diseño al que su imaginación ha llegado con anterioridad» (Wieman 1962:65). Este es el significado más socorrido cuando se identifica a la creatividad con la innovación, y abarca los dos tipos de creatividad de la clasificación de Boden, los sucesos $\mathrm{P}$ y $\mathrm{H}$. Se detecta analizando un producto final (lo que Wieman denomina "bien creado») retrospectivamente hasta llegar a una idea sin precedentes en la mente de un agente que, haciéndola 0 fabricándola, la actualizó.

De acuerdo con Wieman, sin embargo, en el segundo sentido la creatividad es lo que una persona experiencia pero no puede hacer (Wieman 2961:65-66). Su explicación es que detrás de las contingencias de lo que hacen las personas, y de la miscelánea de productos o bienes creados a los que estas acciones dan lugar, está el «bien creativo», que es intrínseco a la propia vida humana en su capacidad de generar personas en relaciones. Este es el tipo de creatividad que no ha empezado aquí, con una idea en la mente, y terminado allá, con un objeto terminado. Por el contrario, ocurre a lo largo, sin principio ni fin. Es, para Wieman, «lo que crea de manera progresiva personalidad en comunidad» (1961:66). Esta es la creatividad de la vida social. Puesto que la vida social no es lo que la persona hace, sino aquello que experiencia: un proceso en el que los seres humanos no crean sociedades, sino que, viviendo en sociedad, se crean a sí mismos y unos a otros (Ingold 1986:202 ss.; Ingold y Hallam 2007:8).

Creo que es justo decir que en líneas generales las ciencias humanas han entendido la creatividad en el primer sentido de los que alude Wieman. Y mi pregunta es: ¿Qué ocurriría si, en lugar de esto, acordáramos dar prioridad al segundo?

Consideremos, por ejemplo, un recién nacido. No se me ocurre un proceso más creativo que aquel por el cual un bebé, como dice el Salmo bíblico, «se entreteje» en el vientre de su madre (Salmo 139.13). Al recibir al recién nacido cuando llega al mundo podríamos decir: «Esto sí es novedoso; ¿acaso habíamos visto algo parecido antes?», o por contra, «Buah, uno más. Los bebés son todos iguales». El último de los comentarios, que nos podría parecer el de una matrona disgustada y cansada, en realidad fue fijado por escrito como una declaración científica por los psicólogos evolucionistas John Tooby y Leda Cosmides. «Los niños», dicen, «son iguales en todos los lugares» (Tooby y Cosmides 1992:33) en el hecho de que llegan al mundo equipados con una arquitectura cognitiva universal preparada para la adquisición de cultura. Pero suponiendo que, al contrario que estos científicos, admitiéramos que este bebé, al menos, fue engendrado creativamente, en lugar de ser una mera réplica extraída del genotipo humano que aún no se ha encontrado con un entorno cultural, ¿daríamos entonces crédito a los felices padres de haber tenido, en el momento de la concepción del bebé, una idea creativa $\mathrm{H}$ sin precedentes, cuya dichosa materialización es acunada ahora ante ellos?

La cuestión es que el crecimiento del bebé en el vientre y, después de su nacimiento, en su infancia y madurez hasta llegar la edad adulta y el envejecimiento, es creativo precisamente en el segundo sentido de Wieman: la creación de la personalidad en comunidad. Esto es, al crecer y ser criadas, las personas experiencian historias de desarrollo y maduración dentro de los campos de relaciones establecidos a través de la presencia de otros y de sus actividades. Y lo que es más importante, este crecimiento no se produce únicamente en términos de fuerza y estatura, sino también en términos de conocimiento, en el empleo de la imaginación y en la formación de ideas. Después de todo, estos últimos son procesos corpóreos de entretejido de materiales y experiencias tanto como lo es el propio desarrollo físico del individuo.

Las ideas también tienen vidas. No emergen completamente formadas de la nada. Una idea es algo parecido a un lugar que visitar. Puedes llegar a ella a través de uno o varios caminos, entretenerte un rato antes de seguir adelante o quizá dar un rodeo y volver a ella un poco más tarde. Cada vez que la revisitas, la idea ha cambiado un poco, enriquecida por los recuerdos y las experiencias de estancias previas. Por lo tanto, haciéndonos eco del famoso aforismo del filósofo Heráclito, no te puedes encontrar dos veces con la misma idea. Esto explica por qué no se pueden revisar los registros del pasado con el objetivo de comprobar si alguien tuvo esa idea anteriormente (y así poder determinar si es de tipo P o H), no es sólo imposible, sino ridículo. No habría ideas, como no habría 
lugares, si no fuera por el movimiento de las personas acercándose a ellas, rodeándolas y alejándose de ellas. Sólo cuando miramos hacia atrás, buscando antecedentes de las cosas nuevas, las ideas aparecen como creaciones espontáneas de una mente encerrada en un cuerpo, en lugar de aparecer como las paradas en el recorrido de los seres humanos moviéndose a través del mundo.

\section{Hacia atrás y hacia delante.}

Lo que falta en la ecuación de la creatividad con la innovación es, por tanto, el crecimiento, el devenir, la formación o creación actual de las cosas, o en una palabra, la ontogénesis. La creatividad en este sentido yace en la capacidad de vivir, la capacidad de las cosas de crecer de manera continuada para superarse a sí mismas. El filósofo británico Alfred North Whitehead denominó a esto la concrescencia auto-superadora (Whitehead 1929:410). En un mundo vivo, defendía Whitehead, no hay sólo cosas concretas, creadas, sino también concrescentes, cosas crecientes. O mejor, se puede mirar al mismo mundo de dos modos: desde el exterior, considerando a cada organismo vivo la encarnación de un diseño evolucionado, o desde dentro, uniéndonos al movimiento generativo de su crecimiento y formación. La distinción de Wieman entre las creatividades, respectivamente, de hacer y experienciar, o entre los bienes creados y el bien creativo, es claramente una versión de la misma cosa.

Ciertamente el paralelismo es poco sorprendente, ya que Wieman había estudiado los textos de Whitehead, y publicado sobre ellos. Antes de esto, había sido un ávido lector de la filosofía de un francés contemporáneo de Whitehead, Henri Bergson. Suenan ecos de Bergson en la idea de una creatividad que puede ser encontrada no en los hechos que distinguen a la persona, sino en la creación de la personalidad en comunidad. «Está... bien decir que lo que hacemos depende de quién somos», escribió Bergson en su Evolución Creadora en 1911, «pero es necesario añadir que somos, hasta cierto punto, lo que hacemos, y que nos estamos creando a nosotros mismos una y otra vez» (Bergson 1911:7). Esta incesante creación de nosotros mismos se corresponde exactamente con la idea de Wieman de una creatividad experienciada más que ejecutada. Es más, tal y como a Bergson le gustaba poner de relieve, el proceso es irreversible. Por lo tanto, entender la creatividad en este sentido es leerla hacia adelante, desde dentro del despliegue de relaciones y procesos que dan lugar a los seres del mundo, más que hacerlo hacia atrás, atribuyendo retrospectivamente los productos finales a los diseños iniciales. Hay que reconocer, con Bergson, que la ontogénesis lleva un tiempo. Y este es el tiempo como duración, no como una sucesión de instantes, sino como la prolongación del pasado hasta el presente. «Duración», escribió Bergson, «es el progreso continuo del pasado que va mordisqueando el futuro y que se expande conforme avanza» (1911:4-5).

Para aquellos de nosotros cuyas vidas están gobernadas en mayor o menor medida por la lógica del capitalismo de bienes y servicios, la identificación de la creatividad con la innovación es algo tan obvio que apenas se cuestiona. Estamos sumidos en esta lógica como consumidores de objetos cuya producción no supuso nada más que la transposición, sobre una materia base, de los diseños que los motivaron. Tampoco la antropología ha permanecido inmune a este modo de pensar (Liep 2001:4). El influyente trabajo de Alfred Gell (1998), Arte y Agencia es un buen ejemplo. El objeto, sostiene Gell, señala la agencia de su creador, quien supuestamente lo ha creado en su mente como una idea, antes de llevar a cabo su materialización. Hacer esta lectura retrospectiva desde el objeto hasta el agente es realizar el acto cognitivo de lo que Gell denomina abducción (1998:13-16). En esto, el autor se deja llevar libremente por el influjo del pragmatista y fundador de la semiótica, Charles Saunders Peirce. A pesar de que los escritos de Peirce sobre el tema son conocidamente oscuros, lo que parecía tener en mente es algo similar a lo que ahora podríamos considerar como "conjeturas fundamentadas». Este es el proceso del detective que, a través de la lectura del rastro de un suceso extraordinario, va siendo inducido hacia la circunstancia o conjunto de circunstancias que resultaron en los hechos observados por mera rutina.

De acuerdo con esta lógica, una obra de arte, en sí misma extraordinaria, lo es únicamente porque puede ser rastreada hasta una concepción sin precedentes en la mente de su creador, de la que todo el trabajo es una continuación inevitable que no tiene nada de especial. Esa lectura retrospectiva, que va desde el objeto a la intención que lo motivó, no reconoce en absoluto los potenciales de generación de formas de las relaciones y los procesos mediante los que las personas y las cosas realmente nacen y crecen. Como reconoció Bergson, entender estos procesos, esto es leer la creatividad hacia adelante, es centrarse en la improvisación en lugar de la abducción (Ingold 2011:216). Es recorrer los caminos a lo largo de los cuales el creador del trabajo va siguiendo a los materiales con los que se ha involucrado. Para los que investigan delitos, el acto está consumado, y el trabajo estriba en deshacer los pasos que lo desencadenaron. Pero si lo que deseamos es entender la creatividad que da lugar a las cosas, tenemos que movernos aguas arriba, desde los hechos hacia su realización. En cierto sentido tenemos que convertirnos nosotros mismos en delincuentes cómplices del delito. 


\section{La creatividad de la imitación.}

Las cosas por tanto, se van sucediendo. Es decir, perduran (Ingold 2013:32). Por ejemplo, como la mayor parte de los chelistas, toco una y otra vez las seis suites de J. S. Bach, escritas para un chelo solista en un intento desolado por interpretarlas bien. Sé que nunca lo conseguiré, puesto que la perfección es una asíntota que ningún músico mortal podrá alcanzar. Pero sigo trabajando en ello. Y conforme lo hago, la música sigue sonando. No es como si el trabajo ya estuviera hecho o acabado en el momento en que Bach acabó de escribir la partitura. Lo que había hecho Bach fue más bien lanzar la música al mundo. $Y$ desde ese momento originario ha continuado fluyendo, avanzando en cada interpretación. En este sentido, el trabajo musical no es creado, sino que es creciente y renaciendo continuamente mientras vive. Es como una corriente incesante y empezar a tocar es empujar tu barca hacia la corriente, sin saber lo que acontecerá. Es una iniciativa plagada de riesgos. Los errores y deslices ocurren, y cuando lo hacen, no hay marcha atrás que los corrija. Sólo podemos rehacernos y continuar con la música.

En este sentido no existe oposición entre la creación y la imitación. Ya he mostrado que no puedes encontrarte dos veces con la misma idea, o revisitar exactamente el mismo lugar, y lo mismo ocurre con la interpretación musical. Nunca puedes repetir exactamente la misma pieza. Es decir, cada interpretación de la pieza puede ser una copia, pero entonces cada copia es un movimiento original, irrepetible. Podríamos decir que la pieza es reproducida en cada ocasión, en el sentido de que se produce una vez tras otra, pero no que es replicada como ocurriría, por ejemplo, si reprodujéramos una grabación con un aparato electromecánico. Lo mismo es cierto para el arte de la caligrafía, que recuerda a la interpretación musical en muchos aspectos. Tal y como el chelista esboza una línea melódica desde la cámara resonante de su instrumento, el calígrafo dibuja sus trazos de tinta desde un cuerpo resonante. A través del pincel, cada matiz e inflexión del gesto manual queda registrado en la línea sinuosa que va abriéndose camino conforme va empapando el tejido del papel. En la ejecución de la caligrafía como en la de la música, no podemos trazar la misma línea dos veces. Y borrarla o volver a tocarla no son una opción.

En su estudio de la influencia de la caligrafía en la sociedad china contemporánea, Yuehping Yenn ha enunciado tres etapas a través de las que se inicia a los principiantes en ese arte. Empiezan por trazar las sombras del modelo a copiar que se sitúa directamente debajo del papel translúcido sobre el que escriben. En la etapa siguiente, el papel y el modelo a copiar están situados uno al lado del otro, forzándolos a improvisar los gestos necesarios por ellos mismos en lugar de guiarse por las sombras del documento maestro. Después, en una etapa final del aprendizaje, se les anima a liberarse del «agarre» de los maestros. En esta etapa «todas las normas aprendidas son apartadas al olvido, y el corazón se convierte en la única guía de la mano» (Yen 2005:123). Sin embargo, los principiantes no dejan de copiar en ningún momento de este proceso formativo en tres pasos. Cada uno de los trabajos de caligrafía es un nuevo «re-pasar», en tanto que está moldeado por los estudios previos, pero cada repaso es en sí mismo un movimiento original que continúa el trabajo, aunque siga caminos que ya han sido recorridos. Este es un movimiento, adoptando la distinción propuesta por el filósofo Gilles Deleuze y el psicoanalista Félix Guattari, no de iteración, sino de itineración (Deleuze y Guattari 2004:410).

Existe un paralelismo significativo entre este proceso de aprendizaje caligráfico y el descrito en un estudio reciente por el antropólogo Eitan Wilf (2012), sobre el aprendizaje con la trompeta de jazz. A pesar de que el contexto institucional (una facultad moderna de Norte América) no podría estar más alejada de un seminario en China, existe el mismo interés por emular las obras maestras del pasado con el objetivo de adquirir un nivel de destreza tal que los practicantes puedan, por así decirlo, desprenderse de las ataduras de su formación previa y lanzarse a por su propio espacio creativo. Donde el principiante en caligrafía copia trabajos en papel, al aprendiz de trompetista se le pide que copie las grabaciones de audio de los solos de los más grandes intérpretes de jazz del pasado. Atendiendo a todos los matices del ritmo, el tono y el timbre, se les instruye para que configuren su interpretación del modelo de la manera más fiel posible. En los mejores casos, deberán ser capaces de tocar el solo en sincronía con la grabación del original de modo que prácticamente sea imposible distinguirlos. En ese momento, la grabación «se desvanece» en la interpretación del estudiante.

La clave de la perspectiva de Wilf estriba en que estos son también momentos de intensa creatividad. No es que el maestro crea y el aprendiz «simplemente» se dedica a imitarlo. Al copiar la interpretación del maestro, el estudiante no sólo lleva a cabo el trabajo, sino que lo habita, sumándose al movimiento y experienciando desde dentro el avance continuo del incipiente sonido cuando está a punto de producirse. A la larga, tal y como describió un profesor, «te acercas tanto al solista que acaba por desvanecerse y te quedas sólo tú» (citado en Wilf 
2012:37). Como en la etapa final del aprendizaje de caligrafía, la fusión definitiva entre la ejecución instrumental del maestro y la del estudiante es una liberación que, de algún modo, permite al estudiante retomar el trabajo donde lo dejó el maestro, guiado únicamente por un corazón que ya late al ritmo del original. Para el trompetista de jazz, y para mí cuando toco a Bach con mi chelo, la música siempre es creciente, nunca creada, siempre en el centro de una corriente incesante que discurre por cada una de las interpretaciones y cada uno de los intérpretes inmersos en ellas. Al introducirse en la corriente, el músico se entrega a una creatividad que no reside en las cosas que hace, sino en lo que experiencia al hacerlo.

La comparación de la música occidental, ya sea «clásica» o «jazz», con la caligrafía oriental nos obliga a reconocer dos aspectos. El primero es que la posibilidad de dedicarnos a lo que Wilf (2012) denomina «rituales creativos», en los que el estudiante puede experimentar una fusión casi mística con el maestro cuyo trabajo está copiando no es, tal como él piensa, únicamente permitida por el registro auditivo. Para Wilf, la música es singular por el hecho de que el texto fuente o modelo que el intérprete intenta emular consiste en un «sonido que se despliega en el tiempo». Con disciplinas como la pintura, pongamos el Guernica de Picasso, podríamos intentar copiar el trabajo, incluso calcarlo sobre el original, pero no podríamos, de acuerdo con Wilf, «experimentar en tiempo real la dimensión de pintar el Guernica en sincronía con el acto de Picasso de pintar el Guernica» (2012:38). Sin embargo, en la práctica caligráfica, en la que, al igual que en la pintura, se emplea lo visual en lugar de lo auditivo, esto es precisamente lo que sucede. El «texto fuente» también se despliega en el tiempo, como lo hacen los gestos manuales que lo producen. Como muestra Yen, no podemos observar un trabajo de caligrafía, por no hablar de entenderlo, únicamente mirándolo. Debemos habitarlo, y reunir nuestra propia visión con la del calígrafo en la producción de sus «trazos de tinta» (Yen 2005:89-90; Ingold 2011:223).

En segundo lugar, no estamos tratando con una simple oposición entre un enfoque de la creatividad «occidental» y «no occidental», ni entre un enfoque «moderno» o «no moderno». La tensión entre «el bien creativo» y «los bienes creados», como señalaría Wieman, o la tensión entre el énfasis retrospectivo en agencia e innovación, y el énfasis prospectivo en el crecimiento y el autodescubrimiento, como nosotros podríamos apuntar, es tan evidente en los contextos educativos occidentales como lo es, incluso en una extensión mayor, en los orientales. Fuyubi Nakamura, por ejemplo, ha mostrado cómo los artistas caligráficos en el Japón de la posguerra han tenido que negociar cada vez más la contradicción entre seguir las líneas trazadas por sus predecesores, a través de una improvisación que responde en cada momento a los caprichos de los materiales que utilizan, y las demandas de nuevos productos que muestren la «creatividad» del artista individual por parte de un mundo del arte mercantilizado (Nakamura 2007).

\section{Siguiendo la partitura.}

Se podría decir que mi elección de ejemplos tomados de la música y la caligrafía suponen una cierta inclinación hacia la interpretación improvisadora. Hasta la música de Bach, aunque haya sido compuesta, deja un gran margen a la interpretación. ¿Qué ocurriría si, por el contrario, la partitura hubiera sido anotada de manera exhaustiva para especificar la interpretación en cada mínimo detalle?. En tal caso, ¿no podría decirse que la pieza existe, como un producto completamente terminado, de manera independiente y como algo previo a cualquier interpretación? ¿Cómo podríamos refutar los argumentos que el antropólogo Dan Sperber aportó con respecto a la preparación de la salsa Mornay en la cocina, diciendo que todo lo que hay que hacer es «convertir en práctica corporal» las detalladas instrucciones o representaciones planteadas en el libro de cocina (Sperber 1996:61). Puede que el músico de repertorio clásico, interpretando una pieza de partitura muy detallada, esté sólo "convirtiendo en práctica» lo que está escrito en el papel. Puesto que no habría nada en la música que no estuviera escrito en la partitura, ¿cómo podría dicha interpretación ser considerada, en sí misma, más creativa que la de una impresora mecánica que imprime una copia de un documento previamente compuesto?.

Estas preguntas plantean una discrepancia entre interpretaciones alternativas de la notación, que podemos denominar respectivamente representacionales y deícticas. Desde el punto de vista de un espectador poco involucrado, podría parecer que la notación representa la pieza de manera completa, en el sentido de que existe una correspondencia unívoca entre las marcas en la página y las prácticas en el mundo real. Sin embargo, para el músico, seguir la partitura o el guión significa corresponder en un sentido bien distinto: el de responder a sus órdenes haciendo uso de destrezas asentadas y relacionadas con la acción y la percepción. Por lo tanto, las instrucciones de un manual de cocina, por ejemplo, o de una partitura musical, no se centran tanto en mapear tus movimientos como en señalar el camino desde el punto en el que estás hasta donde necesitas ir a continuación. Como postes o indicadores en el paisaje, cada uno está situado estratégicamente en las bifurcaciones donde 
podría existir el riesgo de hacer un giro equivocado o pasar de largo el correcto. Entre esos lugares se espera que tú encuentres tu camino, de manera atenta y cuidadosa, pero sin recurrir a regulaciones más explícitas del procedimiento (Ingold 2001:137). Tal como los indicadores orientan al senderista sobre el terreno, la notación proporciona orientaciones a los practicantes para que las sigan mientras vagan a través del campo de prácticas relacionadas que en otra ocasión he llamado «quehacer» (Ingold 2000:195, taskscape en el original).

Un buen ejemplo de esta discrepancia y de los malos entendidos que pueden surgir de ella, está documentado en un estudio reciente de Nicolette Makovicky (2010), relacionado con el encaje de bolillos en una localidad del centro de Eslovaquia. Las encajeras de bolillos usan diagramas especializados de notación efectivamente. Algunas de ellas, movidas por la preocupación de preservar un patrimonio cultural valioso, han empezado a considerar estos diagramas como repositorios de conocimiento tradicional, como si cada uno de estos diagramas fuera una representación codificada de un patrón concreto de labor. Otras sin embargo se resisten a estos esfuerzos de codificación y siguen tratando los diagramas como «herramientas desechables» que ocupan un lugar dentro del trabajo, pero no son anteriores a él, y que pueden ser descartados o redibujados a voluntad conforme éste avanza.

Estas diferencias adquirieron relevancia en la experiencia de una de las interlocutoras de Makovicky, Hana Majerová. En 2007, Majerová trabajaba como profesora en la clase de encaje de bolillos para principiantes en el Centro de Producción de Arte Popular, financiado por el Ministerio de Cultura del país. Allí le pedían que enseñara con diagramas, en los estaban registrados los movimientos y motivos tradicionales que sus estudiantes debían aprender. Sin embargo ella tardó poco en abandonar este enfoque. Para ella, una cosa era que los estudiantes aplicaran las instrucciones de los diagramas y otra bien distinta que desarrollaran las destrezas fundamentales de la labor que les permitirían convertirse en verdaderas profesionales que puedan producir sus propios trabajos de calidad. Este dominio no reside en el repertorio de diseños a disposición del individuo, sino en la maestría en la ejecución de las técnicas básicas de la labor, y en la familiaridad con el uso de las herramientas del oficio y con los movimientos corporales necesarios para manejarlas (Makovicky 2010:85-86).

Frustrada por el enfoque oficial del Centro, Majerová decidió enseñar tal y como ella fue instruida en su momento, en las rodillas de su abuela. Esto originó algunas tensiones con sus superiores, que lo entendieron como un desafío a su autoridad. Sin embargo, era algo completamente coherente con su propia experiencia, en la que, como explicó a Makovicky, realizar diseños y motivos era algo inseparable del trabajo global de trabajar una pieza. Si no le gustaba cómo la pieza estaba evolucionando, descartaría el diseño, y volvería a dibujar partes de él, antes de empezarlo de nuevo. Por tanto, en sus palabras, "Nunca sé cómo terminará hasta que efectivamente ya la he hecho» (citado en Makovicky 2010:91). Entonces, es el proceso de hacer y no el diagrama de notación, lo que determina el producto final. De hecho, como comenta Makovicky, los diagramas «son un resultado material del trabajo de la encajera tanto como el propio encaje» (Makovicky 2010:91). O en palabras del filósofo político Michael Oakeshott, el diseño «es el hijastro, no el padre de la actividad» (Oakeshott 1991:52).

\section{Imaginación y práctica creativa}

La creatividad en el hacer, por tanto, es inherente a la práctica habilidosa que lleva el trabajo hacia adelante, y es también su resultado. Y no a ningún compendio de diseños o representaciones que precedan a la práctica. Pero si esto es así, entonces ¿qué papel juega la imaginación en la práctica creativa? Si la imaginación no tiene que ver con la composición de diseños nuevos antes de su ejecución, en el sentido que le atribuye Wieman de condición para el hacer, ni tampoco tiene que ver con las «ideas creativas» en el sentido Boden de creatividad de tipo $\mathrm{P}$ o de tipo $\mathrm{H}$, entonces, ¿qué es?. En los siguientes párrafos quiero proponer que la imaginación no es una capacidad mental que permite la generación espontánea de ideas, sino una manera de vivir creativamente en un mundo que es en sí mismo creciente y está en continua formación. Imaginar, según el antropólogo Stuart McLean, es "responder creativamente a la creatividad de la incesante autotransformación del mundo" (McLean 2009:231). Esta correspondencia, este responder a un mundo que, en sus relaciones y procesos, también nos responde, es la dinámica generativa que hace que la vida avance, guiándola aspiracionalmente.

En el famoso ensayo sobre la «Historia como sistema» escrito en 1935, el filósofo español José Ortega y Gasset sostenía que apelar a la imaginación en el nombre de nociones tales como el alma, la psique o el espíritu es algo completamente equivocado. Porque al hacerlo se sitúa en el origen de nuestras acciones una conclusión que en realidad nunca se alcanza. En verdad, Ortega escribe, «lo único que nos es dado y que hay cuando hay vida humana es tener que hacérsela» (Ortega y Gasset 1961:200). En esto coincide con Bergson, quien de manera 
similar sostenía, como hemos visto, que la vida es un proceso sin fin de autocreación, o en una palabra, un devenir. Aunque simpatizaba con la postura de Bergson, Ortega fue un paso más allá, insistiendo en que hay algo más que un mero devenir en la tarea específicamente humana de hacerse a sí mismo. Algo más que vivir en hacer la vida (1961:200 fn. 9). Al contrario que otros animales que se convierten en aquello que esté en su naturaleza ser, los humanos deben determinar necesariamente lo que van a ser. La culminación del ser humano está siempre pospuesta, todavía inacabada: un «hombre», dice Ortega es «lo que aún no es» (1961:112-113).

Otra palabra para el «aún no ser» del ser es aspiración. Sin embargo, los humanos no aspiran desde la nada. En cada momento de la vida, se lanzan hacia los horizontes de su conocimiento presente desde lugares ya aprehendidos. Por tanto el movimiento de la vida humana, comparándolo quizá con el de los animales, está estirado temporalmente. Por delante tiene el "aún no» de la aspiración; trae de atrás lo que está "ya ahí» aprehendido. Siendo al mismo tiempo ya y aún no podríamos decir que los humanos están constitutivamente por delante de sí mismos. No se trata de que estén deviniendo en lugar de siendo. Sino de que su devenir está constantemente adelantando su ser. Esto es lo sospechamos cuando decimos, con respecto a nuestra condición humana, no sólo que vivimos nuestras vidas humanas, sino que las llevamos. Y es precisamente en este llevar la vida donde la imaginación cobra un papel relevante.

Recordemos que en la definición de Wieman, «hacer» es actuar conforme a una idea que ya ha sido forjada en la imaginación. La intención o el diseño están ahí antes que el propio acto. «Experienciar», al contrario, supone movernos aguas arriba hacia unos comienzos en los que las ideas todavía han de cristalizar a través del flujo de acción. Como bien creativo, el poder de la imaginación no es la representación mental, tampoco la capacidad de construir una imagen previa al acto de materialización. Es más un impulso generativo de la vida que de manera continuada se adelanta a sí misma. Siguiendo a Ortega, podríamos decir que la imaginación es otra palabra para la aspiración de no ser aún. Y como tal, guía desde delante en lugar de dirigir desde la retaguardia. Pero hacia dónde nos guía no está fijado antes de que empiece la acción. Como se dice coloquialmente, la imaginación tiende a «vagar», tratando de encontrar un camino o improvisar un pasaje. No es su papel determinar un resultado final y cada uno de los pasos que llevan a su consecución. Para Ortega, sin imaginación, sin esta capacidad de precedernos que tenemos, la vida humana sería imposible.

Da la sensación de que en cualquier aventura y en cualquier momento estamos tan preparados como completamente desprevenidos para lo que ha de venir. Nuestra preparación radica en la educación de nuestra percepción. Gracias a ella tenemos práctica en prestar atención y en ser hábiles a la hora de dar una respuesta a lo que ocurre en nuestro entorno (Gibson 1979:254). Mediante esta afinación adquirimos cierta maestría práctica, por ejemplo si caminamos, respondiendo a las irregularidades del suelo, permitiéndonos mantenernos estables en terrenos complicados. Los músicos se convierten en maestros de sus instrumentos, consiguiendo encontrar su camino a través de los pasajes más complicados. Los que se dedican a tejer y a hacer encaje de bolillos adquieren maestría en el trabajo con materiales fibrosos, consiguiendo generar patrones regulares a través de la repetición rítmica de movimientos básicos. Pero con la maestría viene su contrario: la entrega. Como ya hemos visto en el caso de tocar el chelo, lanzarse de lleno a la música es empujar tu barca hacia la corriente de un mundo cambiante, sin saber lo que sucederá. Esto supone experienciar una educación en un sentido bastante distinto, etimológicamente compuesto por ex (fuera) más ducere (guiar) (Masschelein 2010:44). Por tanto ser guiado es ser incitado y arrastrado hacia lo desconocido.

De manera crucial, este movimiento de ex-ducción, de una vida que nos guía hacia un mundo siempre incipiente, siempre a punto de revelarse a sí mismo, no es intencional, sino atencional (Masschelein 2010:48). Cuando decimos de un hecho que está ejecutado intencionalmente, nos referimos a que las acciones externas producidas están moldeadas por un pensamiento interno. La mente propone y el cuerpo dispone. Esto está implícito en la definición de Wieman de «hacer» como la acción que es consecuencia de un diseño preexistente en la mente del agente. Cuando me propongo tocar mi chelo: saco el instrumento de su funda, preparo la música y me coloco en posición; encero el arco. Soy el sujeto y mi interpretación es el predicado. Pero, cuando empiezo a tocar, mi interpretación y yo nos convertimos en una misma cosa, y el hacer da paso al experienciar. Por supuesto, hay aquí una mente activa en la atencionalidad de experienciar. Al igual que la hay en la pintencionalidad de hacer. Pero es una mente inmanente al propio movimiento, más que una causa que origine que ese movimiento sea entendido como un efecto. O resumiéndolo más aún, mientras la intención del sujeto converge hacia un origen, su atención surge de alejarse de él. Este alejamiento, que en vez de posicionarte en un lugar, te saca de él, es bastante literalmente una práctica de exposición (Masschelein 2010:46). Lejos de asegurarnos un lugar en el mundo, nos deja abiertos y vulnerables. 


\section{Acción sin agencia}

¿Cuál es entonces la relación entre la preparación y su ausencia, o entre los modos de educación que residen respectivamente en la afinación y la exposición? Anteriormente sugerí que a diferencia de otras criaturas que viven su vida pero no la guían, las vidas de los seres humanos están temporalmente tensadas entre el ya y el aún no. Entonces, ¿qué guía y qué sigue?. La respuesta habitual es proclamar que los humanos, como seres intencionales, esto es, como agentes, deliberan antes de actuar, en el sentido de Wieman de hacer aquello que ya han imaginado. Es decir, la mente ordena y el cuerpo obedece de una manera más o menos mecánica a sus directrices. Según esto, la maestría es cognitiva: si los humanos guían sus propias vidas es solamente gracias a su capacidad de concebir diseños antes de ejecutarlos, algo que los animales son completamente incapaces de hacer, al menos para una ciencia de la mente construida sobre principios cartesianos. El maestro ajedrecista, por ejemplo, planea los movimientos en su cabeza, mediante computaciones mentales de asombrosa complejidad, mientras que las enactaciones subsiguientes, entre las que se encuentran coger y levantar la pieza de una casilla y transportarla a otra, no podrían ser más sencillas. No requieren destreza alguna y una máquina cualquiera podría hacerlas.

Me gustaría plantear sin embargo que en el proceso de experienciar, la relación de prioridad temporal entre la maestría y la entrega es lo opuesto de lo que se asume en los relatos cognitivos o intencionales del hacer. Aquí la entrega guía y la maestría le sigue: la educación como exposición precede la afinación. Más que tener al mando una mente que sabe lo que quiere y que arrastra tras de sí un cuerpo subordinado, lo que guía desde el frente es una imaginación con aspiraciones que avanza a tientas, improvisando un pasaje a través de un mundo todavía informe. Desde atrás viene la percepción aprehendida que ya se ha acostumbrado a las maneras del mundo y es diestra en observar y responder a sus potencialidades.

Una vida que es guiada se sostiene en la tensión entre la entrega y la maestría, entre la imaginación y la percepción, entre la aspiración y lo aprehendido, y entre la exposición y la afinación. En cada uno de estos emparejamientos, el primer término guía y el segundo le sigue. Pero el guía no es autoritario, sino tentativo. No demanda una obediencia pasiva, sino una dedicación activa. Al empujar mi barca hacia la corriente, invoco a mis poderes perceptivos a estar alerta. Y en cada respuesta descubro que, sin yo saberlo, he estado allí antes, como lo estuvieron mis predecesores desde tiempos inmemoriales. Parece que conozco las amarras sin ser consciente siquiera. Al partir con la corriente, hacia el «aún no», ya sé como se va. Por lo tanto, todo experienciar es recordar. Como ha expuesto el fenomenólogo Bernhard Waldenfels (2004:242), «somos más viejos que nosotros mismos»: detrás de los individuos que estamos a punto de llegar a ser, pero aún no somos, están los individuos que ya somos sin siquiera saberlo. En este continuo e itinerante proceso de devenir lo que fuimos y de haber sido el individuo que llegaremos a ser, no hay límite, ningún punto en el que podamos descubrir alguna naturaleza humana básica que estaba ahí antes de que empezara todo.

Concluyo volviendo al concepto de Wieman de bien creativo: aquello que la personalidad experiencia pero no puede hacer. Este experienciar no es pasivo. Si lo fuera, la entrega seguiría, no guiaría. El experienciar activo, en el cual la entrega es la que ejerce de guía, es un tipo de acción sin agencia. Que sea tan difícil de expresar se debe en gran medida al hecho de que las categorías gramaticales a las que hoy estamos acostumbrados imponen una oposición entre la voz activa y pasiva del verbo, de modo que, tal y como observó Émile Benveniste (1971) en un estudio clásico, la primera de ellas es para la "acción hecha» mientras que la segunda es para la «acción padecida». Sin embargo, como muestra Benveniste, en la historia de las lenguas indoeuropeas la oposición activa/pasiva apareció a través de una descomposición de lo que los antiguos gramáticos griegos denominaban la «voz media». Fue esta descomposición la que situó la agencia en primer plano, separando al actor del hecho. En la voz media, por contra, el actor se mueve dentro del proceso de su acción. "Logra algo», escribe Benveniste, «que se ha ido logrando en él». (Benveniste 1971:149).

Esto es lo que para Wieman significa experienciar de una manera activa. Este experienciar no traduce una imagen mental a un objeto en el mundo. Más bien, como hemos podido apreciar en el caso de Hana Majerová's y el encaje de bolillos, el objeto y la idea surgen juntos en la propia acción. Además esta acción es un acto al que te entregas: tú no lo inicias, pero te corresponde hacerlo. Al abordar la tarea quizá te sorprenda descubrir las capacidades perceptivas y destrezas que pensabas no tener. Pero, ¿de dónde surgió esta cosa que has hecho? No tiene un punto de origen, no se desprende de intención alguna. Lo que experienciamos no está hecho por un autor-agente con un diseño en su cabeza. Es más bien parte de un proceso sin fin de atender y responder en el que, como hemos visto, está inmersa toda la vida humana. Exactamente igual que el «ya» está siempre detrás de nosotros, por muy atrás que queramos ir, el «aún no» escapará siempre por delante, más allá del horizonte de nuestras expectativas. Debemos nuestra existencia completa a lo que ocurrió antes y, puesto que lo que viene 
después nos debe su existencia, al menos en parte, a nosotros, aquello que hacemos no pertenece a nadie: ni a nosotros, ni a los otros, sino a la propia historia, o mejor, a la vida. La creatividad de experienciar, resumiendo, no es otra cosa que la de la propia vida.

\section{Bibliografía}

Benveniste, É. 1971. "Active and middle voice in the verb". In Benveniste, É. (ed.), Problems in General Linguistics. Meek, M.E. (trans.). Coral Gables, FL: University of Miami Press, 145-151.

Bergson, H. 1911. Creative Evolution. Mitchell, A. (trans.). London: Macmillan.

Boden, M. 1990. The Creative Mind: Myths and Mechanisms. London: Weidenfeld and Nicolson.

Deleuze, G. and Guattari, F. 2004. A Thousand Plateaus: Capitalism and Schizophrenia. Massumi, B. (trans.). London: Continuum.

Gell, A. 1998. Art and Agency: An Anthropological Theory. Oxford: Clarendon Press.

Gibson, J.J. 1979. The Ecological Approach to Visual Perception. Boston: Houghton Mifflin.

Ingold, T. 1986. Evolution and Social Life. Cambridge: Cambridge University Press.

Ingold, T. 2000. The Perception of the Environment: Essays on Livelihood, Dwelling and Skill. London: Routledge. DOI: $10.4324 / 9780203466025$

Ingold, T. 2001. "From the transmission of representations to the education of attention". In Whitehouse, H. (ed.), The Debated Mind: Evolutionary Psychology Versus Ethnography. Oxford: Berg, 113-153.

Ingold, T. 2011. Being Alive: Essays on Movement, Knowledge and Description. Abingdon: Routledge.

Ingold, T. 2013. Making: Anthropology, Archaeology, Art and Architecture. Abingdon: Routledge.

Ingold, T. and Hallam, E. 2007. "Creativity and cultural improvisation: An introduction". In E.

Hallam and T. Ingold (eds), Creativity and Cultural Improvisation. Oxford: Berg, 1-24.

Kubler, G. 1962. The Shape of Time: Remarks on the History of Things. New Haven, NJ: Yale University Press.

Liep, 2001. "Introduction". In Liep, J. (ed.), Locating Cultural Creativity. London: Pluto Press, 1-13. The creativity of undergoing 139

Makovicky, N. 2010. 'Something to talk about': Notation and knowledge-making among Central Slovak lacemakers". In Marchand, T.H.J. (ed.), Making Knowledge: Explorations of the Indissoluble Relation Between Mind, Body and Environment. London: RoyalAnthropological Institute, 76-94.

Masschelein, J. 2010. "E-ducating the gaze: The idea of a poor pedagogy". Ethics and Education 5(1): 43-53. DOI: 10.1080/17449641003590621

McLean, S. 2009. "Stories and cosmogonies: Imagining creativity beyond 'nature' and 'culture". Cultural Anthropology 24(2): 213-245. DOI: 10.1111/j.1548-1360.2009.01130.x

Nakamura, F. 2007. "Creating or performing words? Observations on contemporary Japanese calligraphy". In Hallam, E. and Ingold, T. (eds), Creativity and Cultural Improvisation. Oxford: Berg, 79-98.

Oakeshott, M. 1991. Rationalism in Politics and Other Essays. Indianapolis, IN: Liberty Press.

Ortega y Gasset, J. 1961. History as a System And Other Essays Toward a Philosophy of History. New York: W. W. Norton.

Sperber, D. 1996. Explaining Culture: A Naturalistic Approach. Oxford: Blackwell.

Tooby, J. and Cosmides, L. 1992. "The psychological foundations of culture". In Barkow, J.H., Cosmides, L. and Tooby, J. (eds), The Adapted Mind: Evolutionary Psychology and the Generation of Culture. Oxford: Oxford University Press, 19-136. 
Waldenfels, B. 2004. "Bodily experience between selfhood and otherness". Phenomenology and the Cognitive Sciences 3(3): 235-248. DOI: 10.1023/B:PHEN.0000049305.92374.0b

Whitehead, A.N. 1929. Process and Reality: An Essay in Cosmology. Cambridge: Cambridge University Press.

Wieman, H.N. 1961. Intellectual Foundations of Faith. London: Vision Press.

Wilf, E. 2012. "Rituals of creativity: tradition, modernity, and the 'acoustic unconscious' in a U.S. collegiate jazz music program". American Anthropologist 114(1): 32-44. DOI: 10.1111/j.1548-1433.2011.01395.x

Yen, Y. 2005. Calligraphy and Power in Contemporary Chinese Society. London: Routledge Curzon.

\section{Biografía}

Tim Ingold es Professor of Social Anthropology en la University of Aberdeen. Ha realizado trabajo etnográfico entre los Saami y finlandeses de Laponia, y ha escrito sobre medio ambiente, tecnología y organización social en el Círculo Ártico, sobre el rol de los animales en la sociedad humana y sobre ecología humana y teoría evolutiva en antropología, biología e historia. Más recientemente, ha explorado las relaciones entre la percepción ambiental y la práctica hábil. Hoy en día, Ingold escribe y enseña sobre la interfaz entre antropología, arqueología, arte y arquitectura. Su último libro The life of lines se publicó en 2015. 